\title{
PERSPECTIVA ECONÔMICA-FINANCEIRA DA INSTALAÇÃO DE PAINÉIS FOTOVOLTAICOS EM MORADIA NO MUNICÍPIO DE JUAZEIRO DO NORTE- CE: UM ESTUDO DE CASO
}

\author{
Dyesla Leandro de Souza (Universidade Regional do Cariri-URCA) \\ dyeslaleandro@gmail.com \\ Dieferson Leandro de Souza (Secretaria de Educação Básica do Estado do Ceará) \\ diefersonleandro@gmail.com \\ Caio Vinicius de Araujo Ferreira Gomes (Universidade Regional do Cariri-URCA) \\ caioviniciusa2@gmail.com
}

\section{Resumo}

O Brasil passa por uma crise climática e econômica, fatores que influenciam diretamente na taxação das bandeiras tarifárias, impulsionando a população a procurar fontes de energias limpas e renováveis e nessa circunstância a energia solar surge como alternativa viável e econômica. A região Nordeste brasileira apresenta excelentes índices de irradiação, mediante esses fatores, o presente estudo apresenta uma análise quanto à viabilidade econômicafinanceira da instalação de painéis fotovoltaicos em uma residência no município de Juazeiro do Norte, localizada ao sul do Estado do Ceará. O projeto responde de forma positiva aos critérios dos indicadores de análise de investimentos utilizados, sendo exequível nos cenários e condições apresentadas. Esta análise é de cunho exploratório, pautada em um estudo de caso, cotejando com fontes bibliográficas e aprofundamento sobre sistemas fotovoltaicos.

Palavras-chaves: Energia solar. Sistema fotovoltaico residencial. Viabilidade financeira.

\section{Introdução}

Vivemos em um período climático instável, decorrente principalmente por o processo de globalização pelo qual passa a Terra, assim a procura por fontes de energias renováveis e limpas ganham destaque e nesta conjuntura, a energia solar obtém destaque, principalmente por não exigir grande demanda de espaço para a sua instalação e por ser uma fonte inesgotável.

O Brasil apresenta elevadas taxas de incidência solar, as quais aliadas aos fatores citados anteriormente torna-se oportuno o uso dessa fonte de energia. A adesão de painéis fotovoltaicos em residências é crescente, de acordo com dados da Empresa de Pesquisa Energética (EPE) no ano de 2014 a geração fotovoltaica em telhados residências no país foi de $230 \%$.

Embora o território brasileiro apresente elevadas taxas de irradiação solar, é na região Nordeste que predomina as áreas com os melhores percentuais de incidência solar. Conforme 
a EPE (2014), os Estados da região que obtém maior destaque são: Paraíba, Alagoas, Bahia, Ceará, Maranhão, Piauí e Sergipe.

Para o funcionamento efetivo do sistema fotovoltaico é necessário observar a irradiação solar do local, não sendo esse o único fator a ser considerado. É necessário fazer um estudo da viabilidade econômica da instalação do sistema desejado, para com base no resultado, analisar se o projeto é exequível ou não, auxiliando na tomada de decisão sobre a adesão do projeto.

Assim, o presente trabalho busca analisar a viabilidade financeira da obtenção de painéis fotovoltaicos solares, utilizando indicadores de análise de investimento. Aplicando-se os métodos de payback simples e descontado, Valor Presente Líquido (VPL) e a Taxa Interna de Retorno (TIR). A Taxa Mínima de Atratividade (TMA) foi definida através do valor/taxa de remuneração de poupança por meio de dados disponibilizados pelo Banco Central do Brasil.

O estudo tem como base os valores médios mensais de consumo de energia, tarifa repassada pelas concessionárias de energia, valor pago mensalmente pela energia consumida e o orçamente para adesão do projeto, obtido através de um simulador. O orçamente oferece dois valores, o mínimo e o máximo, investigando-os para identificação da viabilidade.

\section{Referencial teórico}

\subsection{Ferramenta de análise de investimento}

\subsubsection{Payback}

O payback simples é um método apurado de forma direta, objetivando encontrar o intervalo de tempo necessário para recuperar o investimento realizado inicialmente, ou seja, o período em que os resultados do fluxo de caixa ganho igualem ao valor investido, sem levar em consideração os ganhos obtidos após o prazo de recuperação. Conforme Ross et al.(2015), em decorrência do payback buscar em especial o prazo de retorno e não preocupar-se com o período posterior, poderá induzir a tomada indevida de decisões se utilizar ao "pé da letra" os resultados obtidos.

Divergente ao payback simples o payback descontado leva em consideração a taxa de desconto (ou Taxa Mínima de Atratividade - TMA), pela qual é determinada de acordo com o interesse do investidor, assim sendo os fluxos de caixa terão descontos por esta taxa em 
relação ao período em que foi determinado. Oportunizando considerar o valor do dinheiro em relação ao tempo o payback descontado torna-se mais aconselhável para a avaliação da viabilidade de um projeto (ROSS et al.,2013).

\subsubsection{Valor presente líquido}

O Valor Presente Líquido é um indicador utilizado para mensurar a exequibilidade econômica de um investimento, levando em consideração o dinheiro em relação ao tempo. Consiste em trazer os fluxos de caixa para a data zero, somando-os ao valor investido inicialmente, utilizando à Taxa Mínima de Atratividade como taxa de desconto.

Segundo Gitman (2010), para utilizar o resultado do Valor Presente Líquido como tomada de decisão para aceitação ou rejeição de um projeto é necessário seguir o seguinte critério: se o valor obtivo for maior (positivo) que $\mathrm{R} \$ 0$ então se aceita o projeto, caso contrário, se o valor for negativo, deve-se rejeitar o projeto.

A obtenção do resultado positivo do Valor Presente Líquido possibilitará a empresa um retorno superior ao investimento inicial, ampliando consequentemente a valorização de mercado da empresa e adicionando riquezas aos investidores, condizentes ao valor do VPL (GITMAN, 2010).

\subsubsection{Taxa interna de retorno}

Consoante com Gitman (2010), a Taxa Interna de Retorno corresponde, possivelmente, a uma das técnicas orçamentarias de capital mais utilizada. Refere-se a um indicador que auxilia o estudo da viabilidade econômica-financeira de um projeto, calculando a taxa de desconto necessária para um fluxo de caixa com que o Valor Presente Líquido iguale-se a zero. Geralmente a taxa encontrada é comparada com a Taxa Mínima de Atratividade seguindo os seguintes critérios:

- $\quad$ TIR > TMA, aceita-se o projeto;

- $\quad$ TIR < TMA, rejeita-se o projeto;

- $\quad$ TIR = TMA, a rentabilidade é nula por isso a decisão fica a critério do investidor.

Em conformidade ao pensamento de Ross et al.(2013), a Taxa Interna de Retorno é relevante pois nos instrui a como calcular os mais complexos retornos de investimento. De acordo com 
Chenço (2012), umas das desvantagens do uso da TIR é ser dependente aos fluxos de caixas, mesmo que não seja diretamente influenciada pela taxa de juros do mercado.

\subsection{Cenário da energia solar no Brasil}

De acordo com a Empresa de Pesquisa Energética (2019) a matriz elétrica brasileira é composta por $83,3 \%$ de energias renováveis, sendo as seguintes: hidráulica $(66,6 \%)$, biomassa $(8,5 \%)$, eólica $(7,6 \%)$ e solar $(0,5 \%)$. Comparando os valores da energia solar nas matrizes elétricas nacionais dos anos de 2018 e 2019, com o percentual de 0,1\%, nota-se um crescimento de $0,4 \%$, entretanto essa fonte renovável não chega a compor $1 \%$ da matriz elétrica brasileira.

O Brasil oferece condições climáticas propicias para a utilização de energia solar, por dispor de elevados índices de irradiação solar, sendo que algumas regiões a capitação de raios solares são maiores. Em conformidade ao pensamento de Silva (2015), o Brasil apresenta um potencial maior para a exploração de energia solar que outros países que se destacam no uso dessa fonte.

Conforme Nascimento (2017), valores de incidência de irradiação solar em países como a Alemanha $\left(900 \mathrm{Wh} / \mathrm{m}^{2}-1250 \mathrm{Wh} / \mathrm{m}^{2}\right)$, Espanha $\left(1200 \mathrm{Wh} / \mathrm{m}^{2}-1850 \mathrm{Wh} / \mathrm{m}^{2}\right)$ e França $(900$ $\left.\mathrm{Wh} / \mathrm{m}^{2}-1650 \mathrm{Wh} / \mathrm{m}^{2}\right)$, chegam a serem inferiores se comparados a qualquer região do território brasileiro $\left(1500 \mathrm{Wh} / \mathrm{m}^{2}-2500 \mathrm{Wh} / \mathrm{m}^{2}\right)$. De acordo com Rella (2017) a insolação recebida por o Brasil excede 3000 horas por ano, apresentando a região Nordeste com valores médios diários de irradiação entre 4,5KWh e $6 \mathrm{KWh}$.

Consoante ao estudo, Tsuruda et al. (2017) destaca que deverá ocorrer um crescimento da presença das fontes renováveis solar e eólica na matriz energética brasileira em um período de 25 anos. Conforme a Nota Técnica DEA 26/14 da Empresa de Pesquisa Energética (2014), a tecnologia fotovoltaica tem destaque entre as demais de geração distribuída de pequeno porte e apresenta-se como a mais propicia a ser introduzida na matriz elétrica nacional no horizonte decenal.

\section{Matérias e métodos}

O presente estudo é de cunho exploratório, desenvolvendo-se por meio de revisão bibliográfica e coleta de dados, assumindo o "papel” de estudo de caso. O objeto em análise 
compreende uma residência com 4 habitantes, localizada do Estado do Ceará e específico no município de Juazeiro do Norte, escolhida por sua localizada em área com alta taxa de irradiação solar.

A realização do orçamento para instalação de painéis fotovoltaicos na residência em estudo, sucedeu através da calculadora simuladora do portal NeoSolar (2020), pelo qual foram cedidas as seguintes informações:

- Local da instalação: Juazeiro do Norte - CE;

- O local tem acesso à rede elétrica;

- Estabelecimento residencial;

- Fornecedor de energia: Enel Distribuição Ceará;

- Tarifa de imposto: 0,74764 R $\$ / \mathrm{KWh}$

- Valor médio pago mensalmente em energia: $\mathrm{R} \$ 138,05$

- Consumo médio mensal: $184 \mathrm{KW} / \mathrm{h}$

Os dados fornecidos anteriormente foram elaborados através dos registros de conta da residência em estudo, compreendendo o intervalo de tempo de fevereiro de 2019 a janeiro de 2020, não levando em consideração a tarifa de iluminação pública. Baseado nos valores obtidos, o simulador forneceu algumas informações relevantes e o seguinte orçamento:

- Estimativa de investimento: R\$ 7.416,75 a R\$ 11.343,77;

- Estimativa de economia mensal: R \$100,50;

- Tamanho do sistema: 1,09KWp;

- Número de módulos: 4 módulos;

- Área necessária: 7,63m²;

- Peso: 103,62 Kg.

A empresa ofereceu garantia de 30 anos de funcionamento do equipamento. Nos valores de investimento não foi adicionado o valor de manutenção em relação ao equipamento, tendo em vista o baixo custo de manutenção. Iremos utilizar o período de 15 anos, que representa metade do tempo da garantia oferecida, para a análise de viabilidade financeira.

\section{Resultados e discussões}

Para a determinação da Taxa Mínima de Atratividade (TMA) utilizou-se a taxa de remuneração de poupança através de dados disponibilizados por o Banco Central do Brasil, a 
taxa de rentabilidade no dia 16/01/2020 dessa aplicação era de $0,2588 \%$ ao mês. Como a análise será realizada em relação há anos, a TMA será 3,11\% ao ano.

O simulador utilizado oferece duas estimativas de investimentos, o mínimo e o máximo, respectivamente $\mathrm{R} \$ 7.416,75$ e $\mathrm{R} \$ 11.343,77$, o valor mensal economizado de $\mathrm{R} \$ 100,50$ permanece constante para os dois valores obtidos ao logo do período de análise dos 15 anos, sendo verificados os cenários apresentados com o intuído de testar a viabilidade econômicafinanceira do projeto.

Para a obtenção dos resultados almejados, inicialmente utilizou-se o investimento mínimo de $\mathrm{R} \$ 7.416,75$ realizando os cálculos de fluxo de caixa, fluxo de caixa descontado, fluxo de caixa acumulado, fluxo de caixa descontado acumulado, Valor Líquido Presente e Taxa Interna de Retorno, posteriormente comparando o resultado da TIR com o da TMA. O mesmo procedimento repetiu-se para o valor máximo de investimento de $\mathrm{R} \$ 11.343,77$. O valor da Taxa Mínima de Atratividade permanece o mesmo para ambos os casos apresentados.

Tabela 1- Fluxo de caixa do investimento mínimo.

\begin{tabular}{|c|c|c|c|c|c|c|}
\hline Ano & Investimento & $\begin{array}{c}\text { Economia } \\
\text { anual }\end{array}$ & $\begin{array}{c}\text { Fluxo de } \\
\text { Caixa }\end{array}$ & $\begin{array}{c}\text { Fluxo de } \\
\text { Caixa } \\
\text { Descontado }\end{array}$ & $\begin{array}{c}\text { Fluxo de } \\
\text { Caixa } \\
\text { Acumulado }\end{array}$ & $\begin{array}{c}\text { Fluxo de } \\
\text { Caixa } \\
\text { Descontado } \\
\text { Acumulado }\end{array}$ \\
\hline 0 & -R $\$ 7.416,75$ & & -R $\$ 7.416,75$ & -R $\$ 7.416,75$ & -R $\$ 7.416,75$ & -R $\$ 7.416,75$ \\
\hline 1 & & $\mathrm{R} \$ 1.206,00$ & $\mathrm{R} \$ 1.206,00$ & $\mathrm{R} \$ 1.169,62$ & $-\mathrm{R} \$ 6.210,75$ & $-\mathrm{R} \$ 6.247,13$ \\
\hline 2 & & $\mathrm{R} \$ 1.206,00$ & $\mathrm{R} \$ 1.206,00$ & $\mathrm{R} \$ 1.134,35$ & $-\mathrm{R} \$ 5.004,75$ & $-\mathrm{R} \$ 5.112,78$ \\
\hline 3 & & $\mathrm{R} \$ 1.206,00$ & $\mathrm{R} \$ 1.206,00$ & $\mathrm{R} \$ 1.100,13$ & $-\mathrm{R} \$ 3.798,75$ & $-\mathrm{R} \$ 4.012,65$ \\
\hline 4 & & $\mathrm{R} \$ 1.206,00$ & $\mathrm{R} \$ 1.206,00$ & $\mathrm{R} \$ 1.066,95$ & $-\mathrm{R} \$ 2.592,75$ & $-\mathrm{R} \$ 2.945,70$ \\
\hline 5 & & $\mathrm{R} \$ 1.206,00$ & $\mathrm{R} \$ 1.206,00$ & $\mathrm{R} \$ 1.034,77$ & -R\$1.386,75 & $-\mathrm{R} \$ 1.910,93$ \\
\hline 6 & & $\mathrm{R} \$ 1.206,00$ & $\mathrm{R} \$ 1.206,00$ & $\mathrm{R} \$ 1.003,56$ & $-\mathrm{R} \$ 180,75$ & $-\mathrm{R} \$ 907,37$ \\
\hline 7 & & $\mathrm{R} \$ 1.206,00$ & $\mathrm{R} \$ 1.206,00$ & $\mathrm{R} \$ 973,29$ & $\mathrm{R} \$ 1.025,25$ & $\mathrm{R} \$ 65,92$ \\
\hline 8 & & $\mathrm{R} \$ 1.206,00$ & $\mathrm{R} \$ 1.206,00$ & $\mathrm{R} \$ 943,93$ & $\mathrm{R} \$ 2.231,25$ & $\mathrm{R} \$ 1.009,85$ \\
\hline 9 & & $\mathrm{R} \$ 1.206,00$ & $\mathrm{R} \$ 1.206,00$ & $\mathrm{R} \$ 915,46$ & $\mathrm{R} \$ 3.437,25$ & $\mathrm{R} \$ 1.925,31$ \\
\hline 10 & & $\mathrm{R} \$ 1.206,00$ & $\mathrm{R} \$ 1.206,00$ & $\mathrm{R} \$ 887,85$ & $\mathrm{R} \$ 4.643,25$ & $\mathrm{R} \$ 2.813,16$ \\
\hline 11 & & $\mathrm{R} \$ 1.206,00$ & $\mathrm{R} \$ 1.206,00$ & $\mathrm{R} \$ 861,07$ & $\mathrm{R} \$ 5.849,25$ & $\mathrm{R} \$ 3.674,23$ \\
\hline 12 & & $\mathrm{R} \$ 1.206,00$ & $\mathrm{R} \$ 1.206,00$ & $\mathrm{R} \$ 835,10$ & $\mathrm{R} \$ 7.055,25$ & $\mathrm{R} \$ 4.509,33$ \\
\hline
\end{tabular}


Com base nas informações obtidas na Tabela 1, ao utilizar a última parcela negativa do fluxo de caixa acumulado e dividir pelo valor do fluxo de caixa do próximo período, encontramos o valor do payback simples constatando que o tempo de retorno do investimento será de 6 anos, 1 mês e 24 dias.

Para encontrar o payback descontando, utiliza-se o último período com valor negativo do fluxo de caixa descontado acumulado dividindo pelo valor referente ao fluxo de caixa descontado do próximo ano, encontrando o período de 7 anos para a obtenção de retorno do investimento.

O Valor Líquido Presente é de $\mathrm{R} \$ 6.104,73$, obtido através da última parcela do fluxo de caixa descontado acumulado. A Taxa Interna de Retorno é de 13,97\%.

O projeto foi analisado no período de 15 anos, os valores de payback simples e o payback descontando foi inferior ao tempo estudado. Analisando o Valor Líquido Presente e a Taxa Interna de Retorno constata-se que ambos apresentam valores positivos, corroborando para a aceitação do projeto.

Tabela 2- Fluxo de caixa do investimento máximo.

\begin{tabular}{|c|c|c|c|c|c|c|}
\hline Ano & Investimento & $\begin{array}{c}\text { Economia } \\
\text { anual }\end{array}$ & Fluxo de Caixa & $\begin{array}{c}\text { Fluxo de Caixa } \\
\text { Descontado }\end{array}$ & $\begin{array}{c}\text { Fluxo de Caixa } \\
\text { Acumulado }\end{array}$ & $\begin{array}{c}\text { Fluxo de Caixa } \\
\text { Descontado } \\
\text { Acumulado }\end{array}$ \\
\hline 0 & $-\mathrm{R} \$ 11.343,27$ & & $-\mathrm{T} \$ 11.343,27$ & $-\mathrm{R} \$ 11.343,27$ & $-\mathrm{R} \$ 11.343,27$ & $-\mathrm{R} \$ 11.343,27$ \\
1 & $\mathrm{R} \$ 1.206,00$ & $\mathrm{R} \$ 1.206,00$ & $\mathrm{R} \$ 1.169,62$ & $-\mathrm{R} \$ 10.137,27$ & $-\mathrm{R} \$ 10.173,65$ \\
2 & $\mathrm{R} \$ 1.206,00$ & $\mathrm{R} \$ 1.206,00$ & $\mathrm{R} \$ 1.134,35$ & $-\mathrm{R} \$ 8.931,27$ & $-\mathrm{R} \$ 9.039,30$ \\
3 & $\mathrm{R} \$ 1.206,00$ & $\mathrm{R} \$ 1.206,00$ & $\mathrm{R} \$ 1.100,13$ & $-\mathrm{R} \$ 7.725,27$ & $-\mathrm{R} \$ 7.939,17$ \\
4 & $\mathrm{R} \$ 1.206,00$ & $\mathrm{R} \$ 1.206,00$ & $\mathrm{R} \$ 1.066,95$ & $-\mathrm{R} \$ 6.519,27$ & $-\mathrm{R} \$ 6.872,22$ \\
5 & $\mathrm{R} \$ 1.206,00$ & $\mathrm{R} \$ 1.206,00$ & $\mathrm{R} \$ 1.034,77$ & $-\mathrm{R} \$ 5.313,27$ & $-\mathrm{R} \$ 5.837,45$ \\
6 & $\mathrm{R} \$ 1.206,00$ & $\mathrm{R} \$ 1.206,00$ & $\mathrm{R} \$ 1.003,56$ & $-\mathrm{R} \$ 4.107,27$ & $-\mathrm{R} \$ 4.833,89$ \\
7 & $\mathrm{R} \$ 1.206,00$ & $\mathrm{R} \$ 1.206,00$ & $\mathrm{R} \$ 973,29$ & $-\mathrm{R} \$ 2.901,27$ & $-\mathrm{R} \$ 3.860,60$ \\
8 & $\mathrm{R} \$ 1.206,00$ & $\mathrm{R} \$ 1.206,00$ & $\mathrm{R} \$ 943,93$ & $-\mathrm{R} \$ 1.695,27$ & $-\mathrm{R} \$ 2.916,67$
\end{tabular}




\begin{tabular}{llllll}
9 & $\mathrm{R} \$ 1.206,00$ & $\mathrm{R} \$ 1.206,00$ & $\mathrm{R} \$ 915,46$ & $-\mathrm{R} \$ 489,27$ & $-\mathrm{R} \$ 2.001,21$ \\
10 & $\mathrm{R} \$ 1.206,00$ & $\mathrm{R} \$ 1.206,00$ & $\mathrm{R} \$ 887,85$ & $\mathrm{R} \$ 716,73$ & $-\mathrm{R} \$ 1.113,36$ \\
11 & $\mathrm{R} \$ 1.206,00$ & $\mathrm{R} \$ 1.206,00$ & $\mathrm{R} \$ 861,07$ & $\mathrm{R} \$ 1.922,73$ & $-\mathrm{R} \$ 252,29$ \\
12 & $\mathrm{R} \$ 1.206,00$ & $\mathrm{R} \$ 1.206,00$ & $\mathrm{R} \$ 835,10$ & $\mathrm{R} \$ 3.128,73$ & $\mathrm{R} \$ 582,81$ \\
13 & $\mathrm{R} \$ 1.206,00$ & $\mathrm{R} \$ 1.206,00$ & $\mathrm{R} \$ 809,91$ & $\mathrm{R} \$ 4.334,73$ & $\mathrm{R} \$ 1.392,72$ \\
14 & $\mathrm{R} \$ 1.206,00$ & $\mathrm{R} \$ 1.206,00$ & $\mathrm{R} \$ 785,48$ & $\mathrm{R} \$ 5.540,73$ & $\mathrm{R} \$ 2.178,21$ \\
15 & $\mathrm{R} \$ 1.206,00$ & $\mathrm{R} \$ 1.206,00$ & $\mathrm{R} \$ 761,79$ & $\mathrm{R} \$ 6.746,73$ & $\mathrm{R} \$ 2.940,00$ \\
\hline
\end{tabular}

Fonte: Os autores (2020)

Utilizando os dados obtidos na tabela 2 e fazendo uso do mesmo procedimento de análise na tabela 1 , pode-se concluir que o período de retorno encontrado para o payback simples irá ocorrer entre o $9^{\circ}$ e o $10^{\circ}$ ano, sendo mais especifico será em 9 anos, 4 meses e 26 dias, e o prazo para o payback descontando é de 11 anos, 3 meses e 19 dias. O Valor Líquido Presente corresponde a $\mathrm{R} \$ 2.940,00$ (positivo) e a Taxa Interna de Retorno é de 6,49\%.

Apesar de apresentar valores menores aos obtidos nos indicadores do investimento mínimo, o projeto com investimento máximo é viável, pois responde de forma positiva aos critérios de aceitação dos indicadores de análise, ou seja, oferece rentabilidade ao investidor.

\section{Conclusão}

Considerando o sol como uma fonte limpa e inesgotável para geração de energia, o território brasileiro, em especial a região Nordeste, dispõe de condições climáticas favoráveis para a exploração e utilização da energia solar. Diante do cenário econômico crítico em que o país passa e a taxação de tarifas elétricas, o uso da energia solar tornou-se uma oportunidade de economia para quem almeja reduzir custos com as concessionárias elétricas

A utilização do sol como gerador de energia elétrica tornou-se realidade em países que desejam buscar fontes não poluentes ao ambiente. Infelizmente no Brasil, o processo de expansão do uso da energia solar é bem tímido, não conseguindo atingir $1 \%$ da representatividade na matriz elétrica brasileira e a situação torna-se mais crítica para a inserção da energia solar fotovoltaica.

Analisando as informações apresentadas no presente estudo pode-se concluir que a adesão do projeto de instalação de painéis fotovoltaicos na residência com as condições apresentadas é viável em ambos os cenários estudados, obtendo-se retorno do investimento antes da metade 
do tempo oferecido como garantia da empresa. A economia mensal é um fator determinante para estudar a viabilidade financeira.

A fim de testar a viabilidade do projeto em outras condições, o valor da Taxa Mínima de Atratividade foi duplicado, passando a ser $6,22 \%$, onde anteriormente era $3,11 \%$, o novo valor foi aplicado no cenário de investimento máximo, respondendo de forma positiva a nova conjuntura. Apresentou retorno antes do horizonte estipulado, o payback descontado ocorre em 14 anos e 7 meses, e o Valor Líquido Presente é de R\$203,15.

Os aspectos econômicos podem ser determinantes para a aceitação do uso da energia fotovoltaica, porém existem alguns fatores relevantes que podem inviabilizar o uso dos painéis ao longo do tempo, tais como: perda de eficiência dos módulos, mudanças climáticas, inclinação dos painéis, compatibilidade elétrica e sujeira acumulada nas placas.

O cenário ambiental é favorecido com a utilização da energia solar. De acordo com NeoSolar (2020), com a utilização dos seus equipamentos em um período de 30 anos estima-se que será possível a redução de $23.042 \mathrm{Kg}$ de gás carbônico na atmosfera, o que seria equivalente a 165 árvores plantadas.

Para tanto, o uso da energia solar ultrapassou os aspectos de cunho financeiro, compondo uma alternativa efetiva para auxiliar na preservação e manutenção do planeta em virtude da crescente e alarmante crise climática que vivenciamos.

\section{REFERÊNCIAS}

BRASIL. Empresa de Pesquisa Energética. Balanço energético nacional 2019: Relatório síntese/ ano base 2018. Rio de Janeiro, 2019. Disponível em: <http://www.epe.gov.br/sites-pt/publicacoes-dadosabertos/publicacoes/PublicacoesArquivos/publicacao-377/topico470/Relat\%C3\%B3rio\%20S\%C3\%ADntese\%20BEN\%202019\%20Ano\%20Base\%202018.pdf>. Acesso em: 24/01/2020.

BRASIL. Empresa de Pesquisa Energética. Nota técnica DEA 19/14: Inserção da geração fotovoltaica distribuída no Brasil - Condicionantes e impactos. Rio de Janeiro, 2014. Disponível em:<http://www.epe.gov.br/sites-pt/publicacoes-dados-abertos/publicacoes/PublicacoesArquivos/publicacao251/topico-311/DEA\%2019\%20\%20\%20Inser\%C3\%A7\%C3\%A3o\%20da\%20Gera\%C3\%A7\%C3\%A3o\%20Fotovoltaica\%20Distribu\%C3\%A Dda\%20no\%20Brasil $\% 20$ -

\%20Condicionantes\%20e\%20Impactos\%20VF\%20\%20(Revisada)[1].pdf\#search=DEA\%2019>. Acesso em: 24/01/2020.

BRASIL. Empresa de Pesquisa Energética. Nota técnica DEA 26/14: Avaliação da eficiência energética e geração distribuída para os próximos 10 anos (2014-2023). Rio de Janeiro, 2014. Disponível em: $<$ http://www.epe.gov.br/sites-pt/publicacoes-dados-abertos/publicacoes/PublicacoesArquivos/publicacao251/topico- 
311/DEA\%2026\%20Efici\%C3\%AAncia\%20Energ\%C3\%A9tica\%20e\%20Gera\%C3\%A7\%C3\%A3o\%20Distri bu\%C3\%ADda\%20para\%20os\%20pr\%C3\%B3ximos\%2010\%20anos\%5B1\%5D.pdf>. Acesso em: 24/01/2020.

CHENÇO, E.C. Fundamentos em finanças. 2012, 1.ed.rev. Curitiba, PR: IESDE Brasil.

GITMAN, L.J. Princípios de administração financeira: tradução Allan Vidigal Hastings; revisão técnica Jean Jacques Salim. 2010.12.ed. São Paulo: Pearson Prentice Hall.

NASCIMENTO, R.L. Energia solar no Brasil: situação e perspectivas. Brasília: Câmara dos Deputados / Consultoria Legislativa/ Estudo Técnico. 2017. Disponível em:

<http://bd.camara.gov.br/bd/bitstream/handle/bdcamara/32259/energia_solar_limp.pdf?sequence=1 >. Acesso em: 24/01/2020.

NEOSOLAR. Calculadora solar fotovoltaica. Disponível em: <https://www.neosolar.com.br/simulador-solarcalculadora-fotovoltaica>. Acesso em: 23/01/2020.

RELLA, R. Energia solar fotovoltaica no Brasil. Revista de iniciação científica. Criciúma, v.15, n.1, 2017. ISSN 1678-7706.

ROSS, S.A.; WESTERFIELD, R.W.; JAFFE, J.F.; LAMB, R. Administração financeira. 2015. 10.ed. Porto Alegre: AMGH. Disponível em: <https://www.studocu.com/fr/document/inseec-alpes-savoie/financial-riskmanagement/autre/362911820-administracao-financeira-stephen-a-ross-pdf-copia/2620368/view>. Acesso em: $25 / 01 / 2020$.

ROSS, S.A.; WESTERFIELD, R.W.; JORDAN, B.D.; LAMB, R. Fundamentos de administração financeira. 2013. 9.ed. Dados eletrônicos. Porto Alegre: AMGH. Disponível em:

<https://www.academia.edu/12772501/LIVRO_Fundamentos_de_Administra\%C3\%A7\%C3\%A3o_e_Finan\%C 3\%A7as_-_Ross>. Acesso em: 25/01/2020.

SILVA, R. M. Energia Solar no Brasil: dos incentivos aos desafios. Brasília: Núcleo de Estudos e Pesquisas/CONLEG/Senado, Fevereiro/2015 (Texto para Discussão nº 166). Disponível em: <https://www12.senado.leg.br/publicacoes/estudos-legislativos/tipos-de-estudos/textos-para-discussao/td166>. Acesso em: 23/01/2020.

TSURUDA, L.K.; MENDES, T.A.; VITOR, L.R.; SILVERA, M.B. A importância da energia solar para o desenvolvimento sustentável e social. In: 6 International Workshop: Advances in cleaner production organizational report. São Paulo, 2017. Disponível em:

<http://www.advancesincleanerproduction.net/sixth/files/sessoes/6B/1/tsuruda_et_al_report.pdf $>$. Acesso em: 24/01/2020. 